\title{
The physics potential of a reactor neutrino experiment with Skipper CCDs: measuring the weak mixing angle
}

\author{
Guillermo Fernandez-Moroni, ${ }^{a, 1}$ Pedro A.N. Machado, ${ }^{a, 2}$ Ivan Martinez-Soler, ${ }^{a, b, c, 3}$ \\ Yuber F. Perez-Gonzalez, ${ }^{a, b, c, 4}$ Dario Rodrigues ${ }^{a, d, 5}$ and Salvador Rosauro-Alcaraz ${ }^{e, 6}$ \\ ${ }^{a}$ Fermi National Accelerator Laboratory, \\ Batavia, IL 60510, U.S.A. \\ ${ }^{b}$ Northwestern University, Department of Physics 8 Astronomy, \\ 2145 Sheridan Road, Evanston, IL 60208, U.S.A. \\ ${ }^{c}$ Colegio de Física Fundamental e Interdisciplinaria de las Américas (COFI), \\ 254 Norzagaray street, 00901, San Juan, Puerto Rico \\ ${ }^{d}$ Department of Physics, FCEN, University of Buenos Aires and IFIBA, CONICET, \\ Buenos Aires, Argentina \\ ${ }^{e}$ Departamento de Física Téorica and Instituto de Física Téorica, \\ IFT-UAM/CSIC, Universidad Autónoma de Madrid, \\ Cantoblanco, 28049, Madrid, Spain \\ E-mail: gfmoroni@fnal.gov, pmachado@fnal.gov, \\ ivan.martinezsoler@northwestern.edu, yf perezg@northwestern.edu, \\ rodriguesfm@df.uba.ar, salvador.rosauro@uam.es
}

ABSTRACT: We analyze in detail the physics potential of an experiment like the one recently proposed by the vIOLETA collaboration: a kilogram-scale Skipper CCD detector deployed 12 meters away from a commercial nuclear reactor core. This experiment would be able to detect coherent elastic neutrino nucleus scattering from reactor neutrinos, capitalizing on the exceptionally low ionization energy threshold of Skipper CCDs. To estimate the physics reach, we elect the measurement of the weak mixing angle as a case study. We choose a realistic benchmark experimental setup and perform variations on this benchmark to understand the role of quenching factor and its systematic uncertainties, background rate and spectral shape, total exposure, and reactor antineutrino flux uncertainty. We take

\footnotetext{
${ }^{1}$ https://orcid.org/0000-0002-1654-9562.

${ }^{2}$ https://orcid.org/0000-0002-9118-7354.

${ }^{3}$ https://orcid.org/0000-0002-0308-3003.

${ }^{4}$ https://orcid.org/0000-0002-2020-7223.

${ }^{5}$ https://orcid.org/0000-0002-7952-7168.

${ }^{6}$ https://orcid.org/0000-0003-4805-5169.
} 
full advantage of the reactor flux measurement of the Daya Bay collaboration to perform a data driven analysis which is, up to a certain extent, independent of the theoretical uncertainties on the reactor antineutrino flux. We show that, under reasonable assumptions, this experimental setup may provide a competitive measurement of the weak mixing angle at few MeV scale with neutrino-nucleus scattering.

Keywords: Neutrino Physics, Precision QED

ARXiv EPrint: 2009.10741 


\section{Contents}

1 Introduction 1

2 Coherent elastic neutrino-nucleus scattering 3

3 Analysis 5

4 Results $\quad 9$

4.1 Quenching factor 10

4.2 Backgrounds 13

$\begin{array}{lll}4.3 & \text { Detector mass } & 14\end{array}$

4.4 Reactor antineutrino flux uncertainty 14

5 Conclusions 15

\section{Introduction}

The recent discovery of coherent neutrino-nucleus scattering (CEvNS) [1] by the COHERENT experiment [2] has stirred a large deal of interest of the high energy physics community. Although experimentally challenging, the CEvNS cross section opens up a new, exciting venue to detect neutrinos. The key reason lies in the fact that this is a relatively large neutrino scattering cross section. This is because when a neutrino transfers a small amount of momentum to a nucleus, with a de Broglie wavelength comparable to nuclear radii, the neutrino probes the nucleus as a whole, instead of scattering with individual nucleons. Therefore, the CEvNS cross section is sensitive to the square of the nucleus weak charge, $\mathcal{Q}_{V}^{\mathrm{SM}} \equiv N-\left(4 s_{W}^{2}-1\right) Z$, where $s_{W} \equiv \sin \theta_{W}$ is the weak mixing angle and $N, Z$ denote the number of neutrons and protons in the nucleus, respectively. This is typically referred to as "coherent enhancement" and may enhance the cross section 100-fold or even more, compared to typical quasi-elastic processes. Such a large enhancement opens up the possibility of employing relatively small detectors and still being able to probe interesting and novel physics in the neutrino sector.

Nevertheless, observing low energy nuclear recoils is still a challenge overcome by only few detectors. Among those are high purity germanium detectors, as used for example in the CoGeNT [3] and GEMMA [4] experiments, and dual phase xenon time projection chambers, such as those used in XENON1T [5]. A more recent and promising technology, and the main focus of the present paper, are Skipper charge couple devices (Skipper CCDs).

Skipper CCDs are 2D pixelated detectors fabricated on high resistivity silicon [6]. Its small pixel size of $15 \mu \mathrm{m}$ by $15 \mu \mathrm{m}$ allows for good spatial resolution and event discrimination. Devices with several millions of pixels can be fabricated with high yield. The high 
resistive substrate permits an active depth of each pixel of $675 \mu \mathrm{m}$ totaling up to approximately 8 grams of active silicon per device. In particular, the great advantage of the Skipper CCD is the unambiguous charge discrimination thanks to its very low readout noise. It provides single-charge counting capability for any ionization packet in the active volume $[7,8]$. The energy resolution is then limited by the silicon absorption process to the different particles. An average energy of 3.749 eVee are needed to ionize an electronhole pair. Extensive measurements of the absorption processes have been studied in these sensors using X-rays [9]. When they are cooled close to liquid nitrogen temperatures the leakage current production is very small [10] and ionizations of few electrons can be unmistakably observed. Pixels are read sequentially by a single or just a few amplifiers per sensor in $\sim 40 \mu$ s per pixel readout which represents a time of approximately 12 millisecond for the single-carrier counting capability reached after doing $\sim 300$ samples). This slow readout implies a poor time resolution since the full readout of a 6 Mega pixels CCD using four amplifiers requires $\sim 5$ hs [11]. The technology is mature enough to allow the integration of many of these sensors running in parallel in a single system. Several groups are already pushing for the integration of many sensors for dark matter experiments: SENSEI experiment (200 grams) [10], DAMIC-M (1kg) [12], and OSCURA (10 kg) [13]. Besides, CCDs have already been recognized as neutrino detectors [14].

The Skipper CCD capability of detecting such low energy nuclear recoils allows these detectors to observe neutrinos emitted from the most intense, known artificial source: commercial nuclear reactors. In nuclear reactors, the fission processes that generate heat (which is afterwards used to generate electricity) always involve copious amounts of neutrino emission. Each of these fission processes emit an average of 6 electron antineutrino and $200 \mathrm{MeV}$ of heat. This roughly translates into $2 \times 10^{20}$ neutrinos per second per $\mathrm{GW}_{t h}$ [15]. To put things in perspective, the Spallation Neutron Source (SNS), the neutrino source used by the COHERENT collaboration, has an intensity of about $5 \times 10^{20}$ protons on target (POT) per day [2]. For SNS energies, each proton delivered on the target should lead to an average of about 0.24 neutrinos originated from the decay of pions produced in the beam. Another example can be made with the future DUNE experiment which plans to deliver $1.1 \times 10^{21}$ POT per year of running, or yet a neutrino flux of $3.4 \times 10^{6} / \mathrm{cm}^{2} / \mathrm{s}$ in the near detector. Despite the enormous neutrino flux, nuclear reactors emit neutrinos with much lower energies than the aforementioned sources, typically around the $\mathrm{MeV}$ scale. A coherent scattering of those neutrinos would transfer not more than a couple hundred electronVolts to the recoiled nucleus. This makes the use of such intense neutrino source very difficult to most detector technologies. Exceptions are the CONNIE [16], NUCLEUS [17] and CONUS [18] experiments. In addition to those, Skipper CCDs are in a special position to leverage nuclear reactors due to their very low energy thresholds.

The physics reach of several CEvNS experiments have been studied in the literature. For example, light mediators coupling to standard model particles have been searched for in the CONNIE experiment [19]. On the theoretical side, there are several studies evaluating the physics capabilities of CEvNS experiments, including the following: CEvNS cross section measurements [20, 21], nuclear physics probes [22], neutrino non-standard interactions [23-37], axion-like particles [38], light mediators and neutrino electromagnetic prop- 
erties [39-49], solar physics [50-52], light sterile neutrinos [53-59] and dark matter/dark sector models [60-63]. Nevertheless, the potential of Skipper CCDs near the core of a commercial nuclear reactor, despite very promising, is still largely unknown. Two main reasons for this stem from the scarcity of measurements on quenching factors at very low energies, and the characterization of backgrounds in these experiments. The latter is particularly important as Skipper CCD readout is relatively slow, making it very hard to implement active vetos to enhance signal to noise ratio.

A detailed study in this context seems very timely, particularly due to the proposal of the recently formed neutrino Interaction Observation with a Low Energy Threshold Array (vIOLETA) collaboration: deploying a 1 to $10 \mathrm{~kg}$ Skipper CCD detector near the Argentinian commercial nuclear reactor Atucha II [64-67]. The present manuscript proposes to perform a study of physics potential, characterizing the relative importance of quenching, background modeling, systematics and statistics. This may serve as a guide to future experiments as it identifies sensitivity bottlenecks and layouts where key improvements may be needed.

To exemplify the physics potential, we choose to analyze the sensitivity to the weak mixing angle. The weak mixing angle is one of the key quantities related to the electroweak symmetry breaking of the standard model, as it relates the mass of the $W$ and $Z$ bosons, and its running in a given renormalization scheme such as $\overline{\mathrm{MS}}$ is an important consistency test of the standard model. This is a challenging measurement which can serve as a proxy to demonstrate sensitivity to low scale new physics scenarios [68]. We expect the determination of $\sin \theta_{W}$ via CEvNS to be one of the major cornerstones in any precision CEvNS experiments [69]. Besides, there is no neutrino measurement of the weak mixing angle at scales below the $\mathrm{GeV}$ that proves to be competitive against other observations.

Finally, in our analysis we propose to use current data from reactor neutrino experiments, specifically Daya Bay [70], to constrain the reactor neutrino flux. This is very relevant given the current unresolved reactor antineutrino anomaly situation: the predicted neutrino fluxes do not agree with data from several experiments. Our method would be more independent of the theoretical calculations and, more importantly, their estimated theory uncertainty.

\section{Coherent elastic neutrino-nucleus scattering}

Coherent elastic neutrino-nucleus scattering is a standard model process in which a neutrino scatters coherently off of a nucleus [1]. If the momentum transfer is low enough, the wavelength of the (virtual) mediator can be comparable to the size of the nucleus. The interaction then probes the nucleus as a whole, not distinguishing among neutrons and protons. Neutral current interactions can be parametrized by the following effective Lagrangian

$$
\mathscr{L}_{\text {eff }}^{Z}=4 \sqrt{2} G_{F} J_{Z}^{\mu} J_{\mu Z},
$$

where the neutral currents $J_{Z}^{\mu}$ are

$$
J_{Z}^{\mu}=\sum_{f=\nu, u, d}\left[\overline{f_{L}} \gamma^{\mu} \tau^{3} f_{L}-\sin ^{2} \theta_{W} \bar{f} \gamma^{\mu} Q_{f} f\right]
$$


Here, $G_{F}$ is the Fermi constant, $\tau^{3}$ denotes the third Pauli matrix, $\sin ^{2} \theta_{W}$ is the weak mixing angle, $Q_{f}$ is the electric charge of the fermions $f$ and the sum runs over $f=\nu, u, d$. In this case, the differential standard CEvNS cross section can be written as

$$
\frac{d \sigma^{\nu}}{d E_{R}} \simeq\left[\mathcal{Q}_{V}^{\mathrm{SM}}\right]^{2} \mathcal{F}^{2}\left(E_{R}\right) \frac{G_{F}^{2} m_{N}}{4 \pi}\left(1-\frac{m_{N} E_{R}}{2 E_{\nu}^{2}}\right)
$$

where $m_{N}$ and $E_{R}$ are the mass and recoil energy of the struck nucleus and $E_{\nu}$ is the incoming neutrino energy. As mentioned in the introduction, the weak charge of the nucleus is defined as

$$
\mathcal{Q}_{V}^{\mathrm{SM}}=N-\left(1-4 \sin ^{2} \theta_{W}\right) Z
$$

where $N$ and $Z$ are the number of neutrons and protons in the target nucleus and $\mathcal{F}\left(E_{R}\right)$ is a form factor that parametrizes the coherence of the interaction. It is important to notice that this cross section is proportional to the square of the number of constituents, such as expected in a coherent scattering, with the modification introduced by the $1-4 \sin ^{2} \theta_{W}$ factor, coming from the different effective weak coupling of protons and neutrons.

The form factor describes the coherence of the interaction. Here, we will focus on the simplistic Helm form factor [40, 71, 72],

$$
\mathcal{F}\left(E_{R}\right)=3 e^{-k^{2} s^{2} / 2}[\sin (k r)-k r \cos (k r)] /(k r)^{3}
$$

where $k \equiv \sqrt{2 m_{N} E_{R}}, s \simeq 1 \mathrm{fm}$ is the nuclear skin thickness, $r=\sqrt{R^{2}-5 s^{2}}$, and $R \simeq 1.14(Z+N)^{1 / 3} \mathrm{fm}$ is the effective nuclear radius. This form factor parametrizes the internal structure of the nucleus as seen by the incoming neutrino. At the MeV scale, $F \approx 1$, which corresponds to a fully coherent interaction.

To have a better understanding of all those quantities, it is useful to plug in numbers relevant to our study into these formulas. We will study the weak mixing angle sensitivity of an experimental setup consisting of a Skipper CCD detector deployed nearby a commercial nuclear reactor. For typical neutrino energies at the $\mathrm{MeV}$ scale, the maximum nuclear recoil achievable for a nucleus $N$ is

$$
E_{R}^{\max }=\frac{2 E_{\nu}^{2}}{m_{N}+2 E_{\nu}} \simeq 76 \mathrm{eV}\left(\frac{E_{\nu}}{\mathrm{MeV}}\right)^{2}\left(\frac{28 m_{p}}{m_{N}}\right)
$$

where $m_{p} \simeq 0.938 \mathrm{GeV}$ is the proton mass. Here we can see the difficulty in detecting CEvNS with reactor neutrinos: the detector needs to be sensitive to energy depositions of $100 \mathrm{eV}$ or so, to be able to take advantage of the large neutrino flux. Conversely, the minimum neutrino energy for a given nuclear recoil is

$$
E_{\nu}^{\min }=\frac{1}{2}\left(E_{R}+\sqrt{E_{R}^{2}+2 E_{R} m_{N}}\right) \simeq 1.1 \mathrm{MeV}\left(\frac{E_{R}}{100 \mathrm{eV}} \frac{m_{N}}{28 m_{p}}\right)^{1 / 2}
$$

Finally, the typical momentum transfer $q$ for the recoil energies of interest is

$$
\sqrt{-q^{2}}=\sqrt{2 E_{R} m_{N}} \simeq 2.4 \mathrm{keV}\left(\frac{E_{R}}{100 \mathrm{eV}} \frac{m_{N}}{28 m_{p}}\right)^{1 / 2}
$$


The form factor is more complicated to write in a useful form. Nevertheless, noticing that

$k s \simeq 1.2 \times 10^{-5}\left(\frac{E_{R}}{100 \mathrm{eV}} \frac{m_{N}}{28 m_{p}}\right)^{1 / 2}$ and $k r \simeq 3.2 \times 10^{-5}\left(\frac{E_{R}}{100 \mathrm{eV}} \frac{m_{N}}{28 m_{p}}\right)^{1 / 2}$ (for silicon)

allows us to expand the form factor for small momentum transfer yielding $\mathcal{F} \sim 1+\mathcal{O}\left(k^{2} s^{2}, k^{2} r^{2}\right)$. Therefore, for this experimental setup, the form factor is very close to $\mathcal{F}\left(E_{R}\right) \sim 1$ and we will approximate it to unity henceforth. Using this approximation, we can approximate the differential cross section by

$$
\frac{d \sigma}{d E_{R}} \simeq 2.3 \times 10^{-38}\left(1-\frac{2 E_{R} m_{N}}{E_{\nu}^{2}}\right)\left(\frac{\mathcal{Q}_{V}^{\mathrm{SM}}}{14}\right)^{2}\left(\frac{m_{N}}{28 m_{p}}\right) \frac{\mathrm{cm}^{2}}{\mathrm{eV}} .
$$

Note however that $\mathcal{Q}_{V}^{\mathrm{SM}}$ depends on the weak mixing angle. The last expression we will present here draws upon the fact that $\sin ^{2} \theta_{W} \simeq 0.238$ is close to $1 / 4$. By writing $\mathcal{Q}_{V}^{\mathrm{SM}}$ around $\sin ^{2} \theta_{W}=0.25(1-\epsilon)$ we obtain

$$
\left[\mathcal{Q}_{V}^{\mathrm{SM}}\right]^{2}=N^{2}\left(1-2 \frac{Z}{N} \epsilon+\frac{Z^{2}}{N^{2}} \epsilon^{2}\right)
$$

For example, from the above formula we expect a $1 \%$ change in $\sin ^{2} \theta_{W}$ to correspond roughly to a $2 \%$ change in the cross section, see figure 1 .

Finally, a few words regarding the weak mixing angle. The definition of the weak mixing angle and its renormalization group running depends on the renormalization scheme. In this work we adopt the $\overline{\mathrm{MS}}$ renormalization scheme, in which the weak mixing angle for very low momentum transfer is predicted to be $\sin ^{2} \theta_{W}\left(Q^{2}\right)=0.23867$ from the measurement of the same angle at the $\mathrm{Z}$ boson pole [73]. In the standard model, $\sin ^{2} \theta_{W}$ does not run considerably below $Q^{2} \equiv-q^{2} \sim(100 \mathrm{MeV})^{2}$ or so. Therefore, we will assume this value in the following as input in order to estimate the sensitivity of our experimental setup to the weak mixing.

\section{Analysis}

The experimental setup we consider here consists of a Skipper CCD detector, deployed at 12 meters from the main core of a nuclear reactor like the Atucha II reactor complex, located at the province of Buenos Aires, Argentina. Throughout our analysis, the detector is assumed to have a fiducial mass of $1 \mathrm{~kg}$. Later, we will comment on the physics case of a $10 \mathrm{~kg}$ detector. The core is assumed to have a thermal power of $2 \mathrm{GW}$, emitting about $\sim 10^{28}$ electron antineutrinos per year. We will assume a data taking period of 3 years.

Regarding backgrounds, it is expected that the Compton interactions from high energy photons and nuclear interactions from high energy neutrons will be the dominant contributions for the recoil energies of interest. Both processes are well predicted by state-of-the-art 


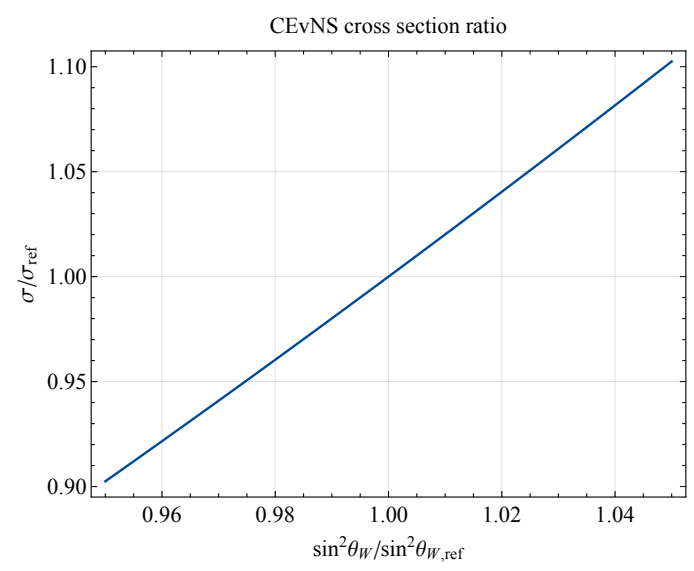

Figure 1. Relative variation of the CEvNS cross section as a function of a change in the weak mixing angle $\sin ^{2} \theta_{W}$. The reference value of the CEvNS cross section $\sigma_{\text {ref }}$ is obtained with $\sin ^{2} \theta_{W, \text { ref }}=0.238$.

particle simulators, but should be analyzed for a specific passive shield configuration to have their impact properly quantified. Therefore, this experimental setup depends crucially on background measurements during reactor-off periods, which are made as short as possible in commercial nuclear reactors. To be realistic, we assume an average reactor-off time of 45 days per year. For the background estimation, we call attention to the measured background in the CONNIE experiment of about $10 \mathrm{kdru}$ (1 differential rate unit, or dru, is 1 event per day-keV-kg) [74]. New studies on the CCD used by CONNIE show that some of its background can be produced by a partial charge collection layer in the back of the sensor [75]. Large charge packets created in this highly doped region are observed as lower energy events since some of the carriers are lost by recombination. This problem can be eliminated by a back side processing of the sensor as explained and tested in the same publication. The CCD detector of CONNIE was located right outside the reactor dome with almost no virtual overburden besides the passive shield. In the scenario considered here, the detector is inside the dome providing some extra reduction to cosmic background. Therefore, we will assume a baseline background of $1 \mathrm{kdru}$, with flat deposited energy spectrum. We will study the impact of higher or lower background rates, as well as the impact of the background spectral shape [76]. Moreover, we will consider the reactor-on background to be negligible, although this needs to be determined by detailed simulations and mandatorily avoided by adding shielding between the detector and the reactor core.

Skipper CCD sensors have shown very low leakage current contributions [77, 78] that allow to explore ionizations up to one or two electrons. Nevertheless we assume a conservative minimum of ionization energy of $15 \mathrm{eV}$, corresponding on average to four ionized electrons. It should be noted that the translation from nuclear recoil energy to ionization energy, which is what is detected by Skipper CCDs, is encoded by the quenching factor. At such low recoils, the quenching factor is not well-known, and measurements to determine it are planned using the Skipper sensors following the procedure presented in ref. [79]. This is critical in reactor neutrino CEvNS, as the neutrino flux grows considerably at lower 
neutrino energies. We will estimate the effect of two different quenching functions and its uncertainties on the experimental sensitivity. Our benchmark will assume the parametrization from ref. [19] of the measurement performed in ref. [79]. As the expected neutrino ionization energy spectrum is fairly broad, and the reconstruction of the ionization energy resolution in Skipper CCDs is excellent (essentially only affected by the silicon absorption, which can be estimated through the fano factor [9]), we do not consider any ionization energy smearing here. Note however that this does not help in reconstructing the incoming neutrino energy. We bin the simulated data in $50 \mathrm{eV}$ ionization energy bins.

Finally, several systematic uncertainties may affect the physics reach of these experiments. Among the most important ones is the uncertainty on the reactor neutrino flux. Several theoretical estimates of this flux have been performed [80, 81]. Although the calculation of these fluxes involve thousands of beta branches and forbidden decays, they achieve a remarkable systematic error of about $2 \%$. Nevertheless, these calculations do not agree with the measured reactor antineutrino flux via the inverse beta decay process, giving rise to a $3 \sigma$ anomaly, dubbed the reactor antineutrino anomaly. The reason for this discrepancy is still unknown, ranging from too aggressive uncertainties [82-84] to new physics beyond the standard model [85-87]. On top of that, theoretical calculations fail to predict a relatively large feature in the reactor neutrino spectrum around neutrino energies of $5 \mathrm{MeV}$ (see e.g. ref. [88]). In view of that, we will estimate the experimental sensitivity to $\sin ^{2} \theta_{W}$ using the flux determination from the Daya Bay experiment [70] instead of relying on theoretical calculations of the neutrino flux and the corresponding systematic uncertainties. Although the former has larger uncertainties, it is a data-driven approach, and thus it is more robust than theoretical estimates. Later, we will show that the flux uncertainty does not play a role as important as other factors such as background rate and quenching factor determination.

The expected signal event rate, for each fuel isotope, measured by the Skipper CCD detector can be obtained by convolving the neutrino flux with the CEvNS cross section, taking into account the quenching factor, namely,

$$
n_{a j}^{q}=\left.\frac{W}{\sum_{q^{\prime}}\left(f_{q^{\prime}} e_{q^{\prime}}\right)} \int_{\text {bin } a} d E_{I} \frac{1}{Q\left(E_{I}\right)}\left(1-\frac{E_{I}}{Q\left(E_{I}\right)} \frac{d Q\left(E_{I}\right)}{d E_{I}}\right) \int_{\text {bin } j} d E_{\nu} \frac{d \phi_{\bar{\nu}_{e}}^{q}}{d E_{\nu}} \frac{d \sigma_{\mathrm{CE} \nu \mathrm{NS}}}{d E_{R}}\right|_{E_{R}=E_{I} / Q\left(E_{I}\right)} .
$$

We denote the ionization energy by $E_{I}$, and the nuclear recoil energy by $E_{R}$. $W$ is the total reactor power. For convenience, we have assigned three indices to $n_{a j}^{q}$. The upper index $q$ indicates the fuel isotope, that is, ${ }^{235} \mathrm{U},{ }^{239} \mathrm{Pu},{ }^{238} \mathrm{U}$ and ${ }^{241} \mathrm{Pu}$. Associated to that is the relative rate per fission $f_{q^{\prime}}$ and energy released per fission $e_{q^{\prime}}$ for isotope $q^{\prime}$. The first lower index corresponds to the ionization energy bin, while the second one refers to the incoming neutrino energy integration interval. Including the latter is important as it allows us to properly take care of flux uncertainties. The quenching function is $Q\left(E_{I}\right)=E_{I} / E_{R}$. Note that the terms in the outer integral come from the Jacobian when changing the integration variable from $E_{R}$ to $E_{I}$. The intervals of integration should be appropriate for the binning and also respect eq. (2.5). The neutrino flux per fission for each isotope is given by $\phi_{\bar{\nu}_{e}}^{q}$. For energies above the inverse beta decay threshold, $E_{\nu}>1.8 \mathrm{MeV}$, we use 
the antineutrino fluxes measured by Daya Bay [70]. In their analysis, Daya Bay uses the theoretical estimates from refs. [80, 81] as inputs for ${ }^{238} \mathrm{U}$ and ${ }^{241} \mathrm{Pu}$ and extracts the fluxes for the two isotopes with the largest relative rates per fission, ${ }^{235} \mathrm{U}$ and ${ }^{239} \mathrm{Pu}$, from their measurements. For energies lower than the inverse beta decay threshold we will use the flux estimate of Vogel and Engel [89]. We will assume an uncertainty of 5\% in the relative rate per fission of the flux for every isotope, similar to the value used by the Daya Bay collaboration [70] in their extraction of the antineutrino spectra. This value comes from the deviation between the theoretical predictions and the isotopic fuel measurements [90].

The sensitivity over $\sin ^{2} \theta_{W}$ is determined by minimizing the following $\chi^{2}$ for the reactor-on running,

$$
\chi^{2}=(\mathbf{d}-\mathbf{t})^{T} \mathbf{C}^{-1}(\mathbf{d}-\mathbf{t})+\sum_{a}^{\text {bins }}\left(\frac{\alpha_{a}^{B}}{\sigma_{a}^{B}}\right)^{2}+\left(\frac{\alpha_{W}}{\sigma_{W}}\right)^{2}+\sum_{q}^{\text {isotopes }}\left(\frac{\alpha_{q}}{\sigma_{q}}\right)^{2}+\left(\frac{\alpha_{Q}}{\sigma_{Q}}\right)^{2} .
$$

There are several quantities in this expression that need to be defined. Let us start with the last four terms which correspond to the Gaussian distributions (also sometimes referred as Gaussian priors) that parametrize the systematic uncertainties of the present experiment. The first systematic is related to the background determination. During reactor-off running, the experiment will determine the background in each bin. The relative statistical uncertainty of this determination is what enters in $\sigma_{a}^{B}$. The second systematic is on the total reactor power $W$. The third systematic corresponds to the relative rate per fission $f_{q}$, which depends on the isotope composition. The last one is on the quenching factor $Q\left(E_{I}\right)$. The $\alpha$ s parameterize the relative deviation of each parameter (pull parameters). The eq. (3.2) will be minimized for each value of $\sin ^{2} \theta_{W}$ according to the profiling method.

The approach adopted here, regarding the determination of the background, is most conservative. In principle, one can also adopt a more aggressive approach. For example, if the background can be well modeled by MonteCarlo simulations, say by a polynomial function, the reactor-off running would determine the uncertainties on the coefficients of such function, which would then be used in eq. (3.2) (the implementation of the pull parameters would change accordingly).

Now we move on to the first term of the $\chi^{2}$. Here, $\mathbf{d}$ is a vector of simulated data obtained by fixing $\sin ^{2} \theta_{W}=0.238$, the SM expected value, while $\mathbf{t}$ are the theory events where $\sin ^{2} \theta_{W}$ is allowed to vary. These vectors can be constructed by summing up all neutrino energies and isotope contributions in the signal rate in eq. (3.1), as well as background rates.

$$
\mathrm{t}_{\mathrm{a}}, \mathrm{d}_{\mathrm{a}}=\sum_{j, q} f_{q} n_{a j}^{q}+B_{a}
$$

where $B_{a}$ is the background in ionization energy bin $a$. Note that the theory prediction, $\mathbf{t}$, should also properly include the pull parameters $\alpha$. To do so, the following substitutions should be made

$$
f_{q} \rightarrow\left(1+\alpha_{q}\right) f_{q}, \quad B_{a} \rightarrow\left(1+\alpha_{a}^{B}\right) B_{a}, \quad W \rightarrow\left(1+\alpha_{W}\right) W, \quad Q\left(E_{I}\right) \rightarrow\left(1+\alpha_{Q}\right) Q\left(E_{I}\right) .
$$

Note that the relative rate per fission should sum up to 1 , and therefore $\sum_{q}\left(1+\alpha_{q}\right) f_{q}=1$. For the reference values we use $f_{235}: f_{238}: f_{239}: f_{241}=0.55: 0.07: 0.32: 0.06$. 


\begin{tabular}{|l|c|c|c|c|}
\hline Systematic & Background & Reactor power & Relative rate per fission & Quenching \\
\hline Symbol & $\sigma_{a}^{B}$ & $\sigma_{W}$ & $\sigma_{q}$ & $\sigma_{Q}$ \\
Value & $1.2 \%$ & $0.5 \%$ & $5 \%$ & $0-25 \%$ \\
\hline
\end{tabular}

Table 1. Systematic uncertainties used in our analysis. See text for details.

The covariance matrix $\mathbf{C}$ parametrizes correlated and uncorrelated systematic uncertainties in the determination of the flux, as well as statistical uncertainties. We adopt ( $\delta_{a b}$ being the Kronecker delta)

$$
\mathbf{C}_{a b}=\mathrm{d}_{a} \delta_{a b}+\sum_{i j}^{\text {energies }} \sum_{q}^{\text {isotopes }} n_{a j}^{q} n_{b k}^{q} V_{j k}^{q},
$$

where statistical ${ }^{1}$ and systematic uncertainties are encoded in the first and second terms, respectively. Note that the Daya Bay collaboration only provides this correlation matrix for the isotopes with the largest rate per fission, namely, ${ }^{235} \mathrm{U}$ and ${ }^{239} \mathrm{Pu}$, and for neutrino energies above $1.8 \mathrm{MeV}$ (which is the threshold for inverse beta decay). In this case, $V_{i j}^{q}$ are taken from Daya Bay's flux covariance matrix [70] and correlates the deviations of the spectrum at different energies. ${ }^{2}$ For the flux below $1.8 \mathrm{MeV}$, we use a single neutrino energy bin, and we assume an uncorrelated spectral uncertainty of $5 \%$. Moreover, for the subdominant rate per fission of the isotopes ${ }^{238} \mathrm{U}$ and ${ }^{241} \mathrm{Pu}$, we use a $5 \%$ bin-to-bin uncorrelated systematic uncertainty on the flux. Given that the relative rate per fission of these isotopes are assumed to be 0.07 and 0.06 , such systematic uncertainties play a small role in the analysis.

The numerical values for the priors used can be found in table. 1. Note that the background uncertainty is derived from the reactor-off period. We assume the background rate to be $1 \mathrm{kdru}$, flat in ionization energy, an exposure of 45 days per year, for 3 years, and a detector mass of $1 \mathrm{~kg}$. This yields a statistical determination of the background rate of $1.2 \%$ in each $50 \mathrm{eV}$ ionization energy bin.

\section{Results}

We now proceed to the results of our simulation. Our benchmark setup is summarized in table 2. We assume the quenching factor to be the one measured in ref. [79] and parametrized in ref. [19]. The quenching models and the impact of systematic uncertainties will be discussed in detail below. The signal and background event rates spectra for this benchmark are presented in the left panel of figure 2. We will perform studies on variations of this scenario to understand the role of different aspects on the experimental sensitivity. The

\footnotetext{
${ }^{1}$ Notice that, in the absence of systematic uncertainties, the inverse of the covariance matrix corresponds to $\mathbf{C}_{a b}^{-1}=\frac{\delta_{a b}}{d_{a}}$, so that in eq. (3.2) one recovers the standard $\chi^{2}$ for a poissonian statistical uncertainty, $\sigma_{a}=\sqrt{d_{a}}$.

${ }^{2}$ In the covariance matrix, we consider the corrections in the nuclear fuel, the non-equilibrium isotopes, and non-linear isotopes.
} 


\begin{tabular}{|l|c|}
\hline Detector mass & $1 \mathrm{~kg}$ \\
\hline Distance to reactor core & 12 meters \\
\hline Core thermal power & $2 \mathrm{GW}_{\text {th }}$ \\
\hline Exposure & 3 years \\
\hline Reactor off time & 45 days per year \\
\hline Background & $1 \mathrm{kdru}$, flat in $E_{I}$ \\
\hline Quenching factor & Chavarria $[79]$ \\
\hline
\end{tabular}

Table 2. Benchmark experimental setup considered here.

sensitivity to the weak mixing angle in our benchmark scenario is shown in the right panel of figure 2 for two different assumptions on the quenching factor which will be discussed below, the Lindhard and the Chavarria models, without systematic uncertainties on the quenching. Together with our estimate, we also present current measurements (taking into account the running of $\sin ^{2} \theta_{W}$ ) [73] and a forecast for the measurement on the DUNE near detector complex [91].

The precision on $\sin ^{2} \theta_{W}$ achieved by this benchmark setup is $1.4 \%$ for Lindhard and $2.8 \%$ for Chavarria quenching, at mean momentum transfers $\left\langle Q^{2}\right\rangle$ of $4.3 \mathrm{MeV}$ and $6.6 \mathrm{MeV}$, respectively. The mean momentum transfer is different because the minimum detectable recoil energy depends on the quenching. As we can see, under reasonable assumptions, this experimental setup would determine the weak mixing angle with a precision similar or slightly worse than atomic parity violation (APV), and comparable precision to several other determinations, including the future DUNE experiment [91]. The competitiveness of such a small experiment is quite remarkable. The measurement proposed here would be one of the very few determinations of the weak mixing angle with neutrinos, which is particularly important given the discrepancy between measurement and theory prediction observed in the NuTeV experiment [92]. The fact that we observe such a deviation only in the measurement of the weak mixing angle with neutrinos could be an indication of a new interaction in the neutrino sector (see, e.g. [93, 94]). As a side comment, by using the LEP determination of the weak mixing angle and the theoretical prediction for the reactor antineutrino flux, this setup could probe the reactor anomaly at the $3-6 \%$ precision. To estimate how robust this measurement is and how it depends on our assumptions, we proceed to a study of how each ingredient affects our analysis. We will study the role of quenching factor parametrization and uncertainties, as well as background rate and shape.

\subsection{Quenching factor}

As mentioned before, the quenching factor characterizes the fraction of the recoil energy transformed into observable ionization energy. Measurements of the quenching factor in the case of silicon have been previously performed [79]. Low energy measurements disagree with the theoretical prediction obtained using the Lindhard model [105] for the quenching. Given this discrepancy, we will study two cases for the quenching factor. 

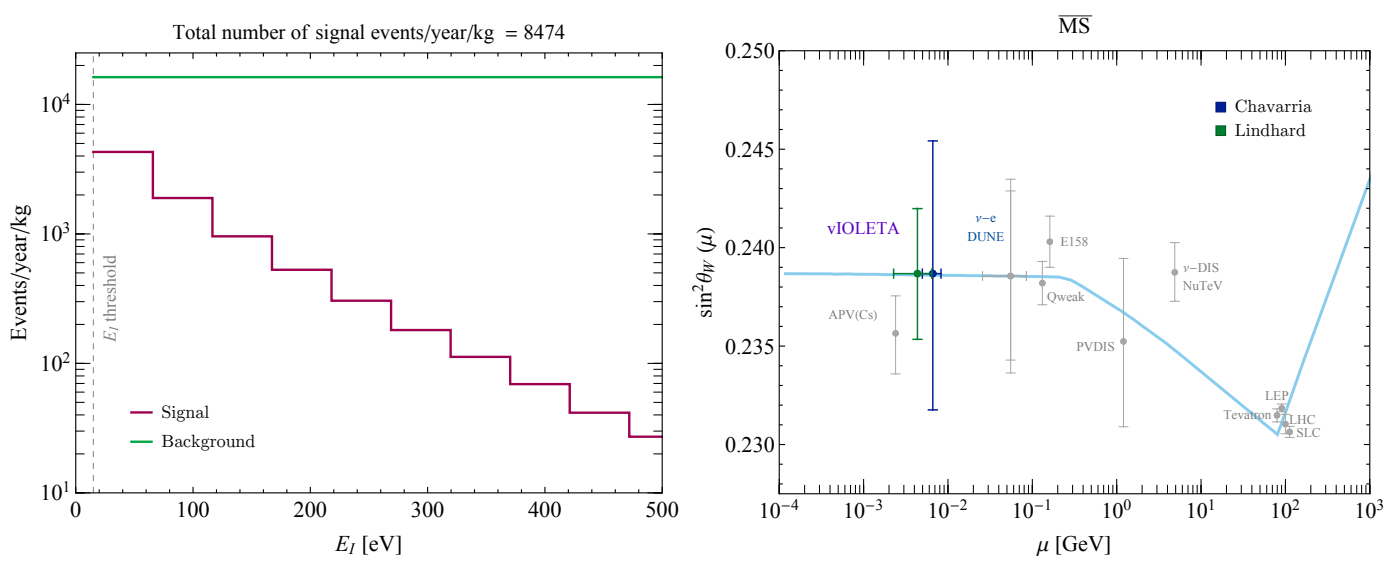

Figure 2. Left: spectral rate of signal (red histogram) and background (green) events for our benchmark setup (see table 2). The vertical dashed line shows the ionization energy threshold of Skipper CCDs. Right: sensitivity of our experimental setup to $\sin ^{2} \theta_{W}$ compared with different experiments (Atomic Parity Violation (APV) [95], Qweak [96], E156 [97], Parity Violation in Deep Inelastic Scattering [98], NuTeV [92], LEP [99], SLC [100], Tevatron [101], LHC [102-104]) in the $\overline{\mathrm{MS}}$ renormalization scheme prediction (light blue line). The DUNE point corresponds to the sensitivity expected measuring neutrino-electron elastic scattering [91]. The two colored data points correspond to two different quenching factors, Lindhard (green) and Chavarria (dark blue). No uncertainty on the quenching was considered. The renormalization scale $\mu$ can be conveniently identified as the average momentum transfer $\mu^{2}=\left\langle Q^{2}\right\rangle$.

Lindhard model. We start by describing the implementation of the widely used Lindhard model for the quenching factor at low energies. We perform a polynomial fit to the Lindhard quenching model given in ref. [106], obtaining the following functional form

$$
Q\left(E_{I}\right)=a_{0}+a_{1} E_{I}+a_{2} E_{I}^{2}+a_{3} E_{I}^{3}+a_{4} E_{I}^{4}+a_{5} E_{I}^{5}+a_{6} E_{I}^{-1}+a_{7} E_{I}^{-2},
$$

where $a_{0}=0.173, a_{1}=0.307 \mathrm{keV}^{-1}, a_{2}=-0.856 \mathrm{keV}^{-2}, a_{3}=1.684 \mathrm{keV}^{-3}, a_{4}=-1.801 \mathrm{keV}^{-4}$, $a_{5}=0.783 \mathrm{keV}^{-5}, a_{6}=5.9 \times 10^{-4} \mathrm{keV}$ and $a_{7}=3.3 \times 10^{-6} \mathrm{keV}^{2}$. Note that, within this parametrization, the Skipper CCD threshold of $E_{I}^{\min }=15 \mathrm{eV}$ would translate to a recoil of $E_{R}^{\min }=E_{I}^{\min } / Q=65 \mathrm{eV}$. From eq. (2.5), the minimum neutrino energy to produce such recoil is about $0.9 \mathrm{MeV}$. Therefore, assuming the Lindhard quenching model, a large portion of the reactor antineutrino flux, below the inverse beta decay threshold, can lead to observable recoils in our experimental setup.

Chavarria model. The measurements performed to determine the quenching factor at low energies [79] can be parametrized by the following ratio of polynomials

$$
Q\left(E_{I}\right)=\frac{p_{3} E_{I}+p_{4} E_{I}^{2}+E_{I}^{3}}{p_{0}+p_{1} E_{I}+p_{2} E_{I}^{2}}
$$

A fit to the data determines $p_{0}=56 \mathrm{keV}^{3}, p_{1}=1096 \mathrm{keV}^{2}, p_{2}=382 \mathrm{keV}, p_{3}=168 \mathrm{keV}^{2}$ and $p_{4}=155 \mathrm{keV}$. This parametrization, which we will refer to as the Chavarria model, has been used by the CONNIE experiment in beyond standard model physics searches [19]. 


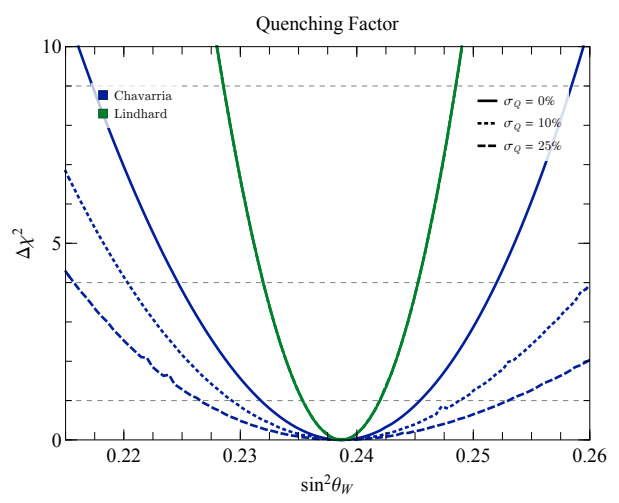

Figure 3. Benchmark sensitivity to $\sin ^{2} \theta_{W}$ for Lindhard (green) and Chavarria (dark blue) quenching factor models, for systematic uncertainties of $0 \%$ (solid), $10 \%$ (dotted) and $25 \%$ (dashed). We indicate $1 \sigma, 2 \sigma$, and $3 \sigma$ precision $\left(\Delta \chi^{2}=1,4,9\right)$ by the gray dashed horizontal lines.

Given that the quenching factor has only been measured down to ionization energies of about $60 \mathrm{eV}$ and the measurement is not in agreement with the theoretical predictions of Lindhard, it is not completely clear what should be assumed for the quenching below that energy. Thus, we use this parametrization as an alternative to the Lindhard quenching factor described above.

One important feature of the Chavarria quenching is that, for small enough $E_{I}$, the recoil energy $E_{R}=E_{I} / Q$ becomes constant. Therefore, it becomes very hard to detect low recoils, as the ionization energy shrinks very quickly for small $E_{R}$. Plugging in numbers, the $15 \mathrm{eV}$ ionization energy threshold translates into a recoil energy of $425 \mathrm{eV}$, which in turn gives us a minimum neutrino energy of $2.3 \mathrm{MeV}$, see eq. (2.5). Thus, compared to the Lindhard quenching, the Chavarria quenching will significantly decrease statistics. Besides, a systematic uncertainty on the Chavarria quenching will change the minimum neutrino energy considerably and therefore significantly affect the rate of signal events. We emphasize however that this assumption about the quenching is very conservative and future measurements will clarify the quenching below $60 \mathrm{eV}$ of ionization energy.

Quenching factor systematics. To understand the impact of systematic uncertainties we evaluate the $\sin ^{2} \theta_{W}$ sensitivity under several assumptions and present the results in figure 3. The assumptions regarding quenching and its systematic uncertainties are the following: Lindhard (green) or Chavarria (dark blue) models, with 0\% (solid), 10\% (dotted) and $25 \%$ (dashed) overall normalization uncertainties in the quenching (note that for Lindhard these lines are all on top of each other). As discussed, the Lindhard quenching yields a considerably superior result compared to the Chavarria model due to statistics. Moreover, in the Lindhard model a variation in the quenching does not change the rate of signal events by much, due to the very low thresholds of Skipper CCD detectors. For any of the quenching uncertainties, the Lindhard quenching would allow for a $1.4 \%$ precision measurement of the weak mixing angle. For the more conservative quenching model of Chavarria, we find that the precision on the mixing angle is significantly decreased to be $2.8 \%, 4.2 \%$ and $6.0 \%$ for a $0 \%, 10 \%$ and $25 \%$ systematic uncertainty, respectively. This 
dramatic effect is simply due to the fact that variations in the quenching lead to significant variations in the signal rates, as much more or much less neutrino flux becomes detectable. We conclude, therefore, that the determination of the quenching factor at low ionization energies is crucial for the physics case of an experiment measuring CEvNS from reactor neutrinos with a Skipper CCD detector.

\subsection{Backgrounds}

In CEvNS, unless the recoiling nucleus is tracked and reconstructed, which is extremely challenging, the typical experimental signature is quite simple: just a small deposition of energy in the detector. Because of that, background rejection is a difficult task in CEvNS detectors. This is particularly true for Skipper CCDs. Due to their slow readout, not even active vetos may be used to reject the cosmic induced background. Background rates and measurements are therefore crucial to the success of the experimental setup considered here. In particular those backgrounds that are correlated with the reactor power. The most intense background generated at the reactor are gammas and thermal neutrons. Gammas are well absorbed by the reactor shield and experiments like CONNIE have reported no excess on electromagnetic shower flux between on and off periods looking at the X-ray florescence of the surrounding materials of the sensors [19]. On the other hand, thermal neutrons do not produce an observable signal on the sensor. Specific gammas and neutron detectors should be used to monitor these and other sub-dominant contributions (like higher energy neutrons) to avoid systematics between reactor on and off periods and input the particle simulators. We will quantify this statement in the following by analyzing the dependence of the experimental sensitivity on the background rate, modeling, and shape. Our benchmark assumes reactor-off running time of 45 days per year which serves to determine the background rate. In the background determination, we simply use the uncorrelated bin-to-bin statistical uncertainty from the reactor-off data taking period. A background model could significantly improve the experimental sensitivity, but that would require a dedicated background study, which is beyond the scope of this paper. For concreteness, we will use the Chavarria quenching as it will result in a more conservative sensitivity estimate, but without quenching systematic uncertainty.

First, we investigate the effect of increasing or decreasing the background rate. In the left panel of figure 4, we present relative $1 \sigma$ determination of $\sin ^{2} \theta_{W}$ as a function of the total background rate for both Chavarria (blue line) and Lindhard (green line) quenching factors. We observe that, in the case of Chavarria quenching, the measurement of the weak mixing angle is highly sensitive on the total background. For instance, if the background is $10 \mathrm{kdru}$, that is, ten times larger than in our benchmark scenario, the precision on $\sin ^{2} \theta_{W}$ gets worse from $2.8 \%$ to $8 \%$, while for $0.1 \mathrm{kdru}$ background rate the precision is enhanced to $1.6 \%$. The dependence on the total background rate is less pronounced for Lindhard quenching, as the signal rate in this case is much higher, and therefore the experimental sensitivity is less affected by backgrounds around $1 \mathrm{kdru}$.

To see how the shape of the background changes the sensitivity, in the right panel of figure 4, we perform the analyses for three representative spectral shapes in ionization energy: flat (black), proportional to $E_{I}^{-1}$ (dashed dark cyan), and linear in $E_{I}$ (dotted 

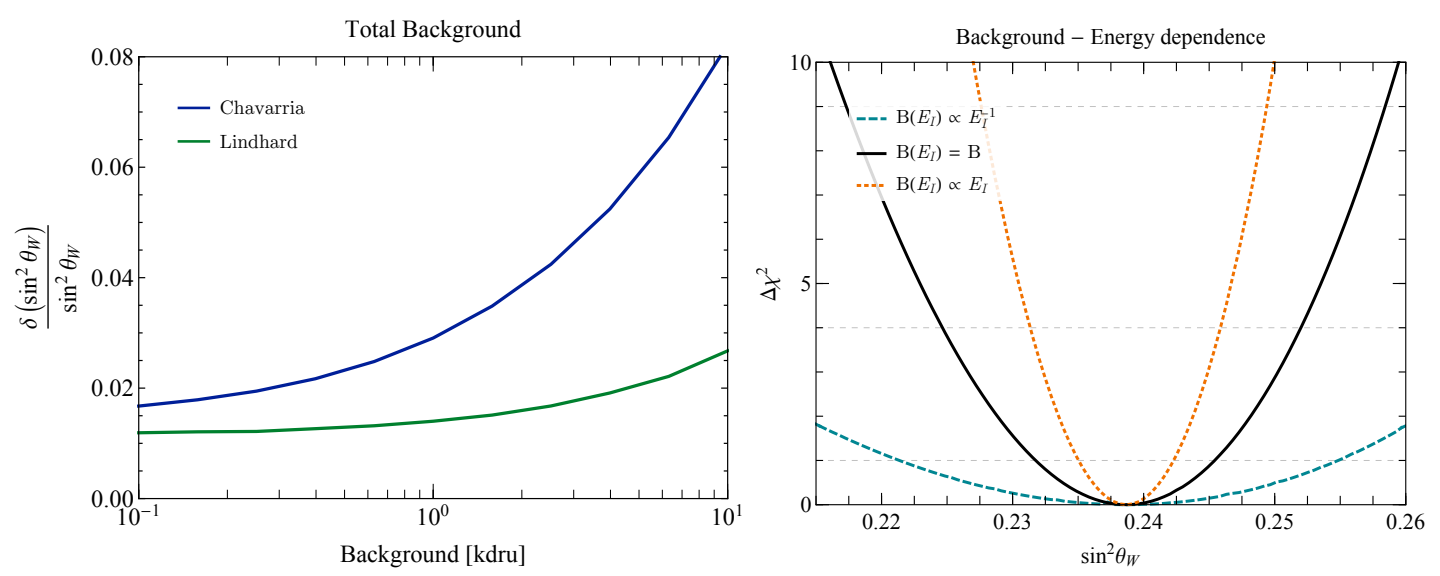

Figure 4. Left: precision on the determination of $\sin ^{2} \theta_{W}$ as a function of background rates, assuming a flat background spectrum, for Chavarria (blue) and Lindhard (green) quenching scenarios. Right: benchmark sensitivity to $\sin ^{2} \theta_{W}$ for different background spectral shapes: flat (black solid), $E_{I}^{-1}$ (dark cyan dashed) and $E_{I}$ (dotted orange).

orange). In doing these studies, we have kept the background rate fixed at $1 \mathrm{kdru}$ in the energy range $E_{I} \in[15,675] \mathrm{eV}$. As already pointed out in ref. [76], if the background grows at low energies, the experimental sensitivity is significantly decreased. This is because in that case, the background has a shape very similar to the signal, see figure 2. Conversely, if the background shrinks at low ionization energy, the experimental sensitivity would be increased. From this, we can appreciate the relevance of properly modeling and mitigating the background rate in experiments employing Skipper CCD detectors.

\subsection{Detector mass}

We also perform a study the dependence of the $\sin ^{2} \theta_{W}$ determination as a function of the total exposure. This helps to understand if the sensitivity is systematic or statistically limited. In figure 5 , we show, for our benchmark scenario, how the $1 \sigma$ uncertainty on $\sin ^{2} \theta_{W}$ depends on the total exposure for Chavarria (blue line) and Lindhard (green line) quenching. In the first case, going from $3 \mathrm{~kg}$-year to $30 \mathrm{~kg}$-years of exposure, the precision on the weak mixing determination could go from $2.8 \%$ to $0.9 \%$ evidencing that the experiment sensitivity is not systematics limited, and thus a larger detector or longer exposure time would yield significantly better physics measurements. We observe a similar pattern for the Lindhard quenching case.

\subsection{Reactor antineutrino flux uncertainty}

As mentioned above, in view of the recent reactor antineutrino anomaly, one may find more robust to do precision analysis with the Daya Bay flux determination. Nevertheless, it is a useful exercise to see the dependence of the sensitivity to $\sin ^{2} \theta_{W}$ on the reactor antineutrino flux uncertainty. To do so, we perform our last analysis in this manuscript under three assumptions regarding the flux uncertainties, in figure 6: Daya Bay covariance matrix [70] (solid blue), $2 \%$ theory normalization error [80, 81] (dotted blue) and $5 \%$ flux 


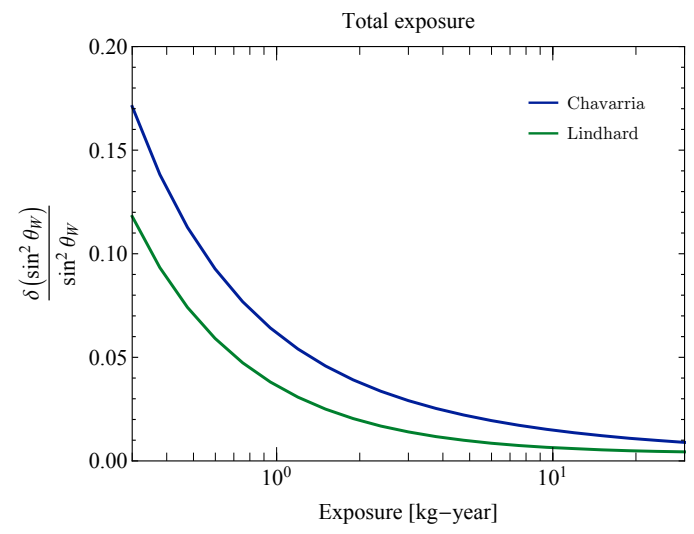

Figure 5. Precision on the determination of $\sin ^{2} \theta_{W}$ as a function of the total exposure for Chavarria (blue) and Lindhard (green) quenching scenarios.

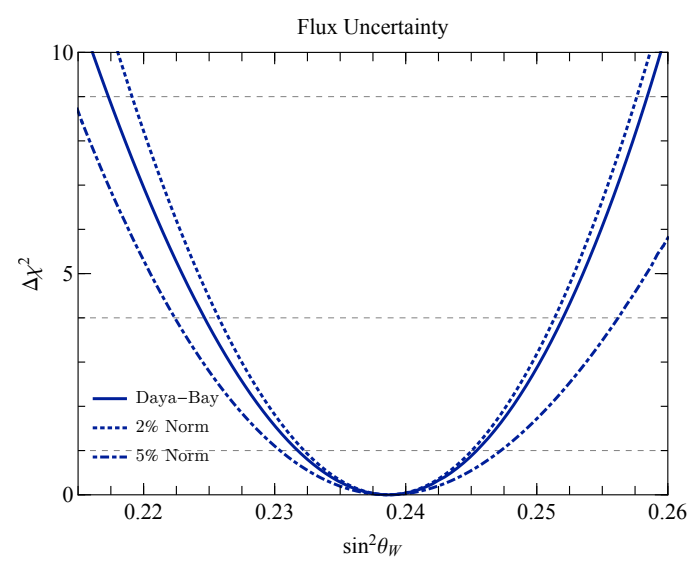

Figure 6. Precision on the determination of $\sin ^{2} \theta_{W}$ for different flux uncertainties: Daya Bay covariance matrix [70] (solid blue), $2 \%$ theory normalization error [80, 81] (dotted blue) and 5\% flux normalization error [82-84] (dashed blue).

normalization error [82-84] (dashed blue). As we can see, the Daya Bay flux determination is already quite good for our benchmark sensitivity. By reducing the flux uncertainty from Daya Bay's covariance matrix to a $2 \%$ overall normalization, the sensitivity improves marginally. We therefore conclude that, although an improved flux model or determination would be beneficial, this is not a bottleneck in our experimental proposal.

\section{Conclusions}

In this paper, we have analyzed the sensitivity to the weak mixing angle of an experimental configuration like the recently proposed vIOLETA experiment, a Skipper CCD detector deployed 12 meters away from the core of the commercial nuclear reactor Atucha II in the province of Buenos Aires, Argentina. We have analyzed the impact of several experimental aspects on the estimated sensitivity: quenching factor and its uncertainty, background rate and spectral shape, and total exposure. We have quantified the crucial role of the quenching 
factor on the sensitivity. Nevertheless, the role of systematic uncertainties in the quenching depends on the quenching itself. For the Lindhard quenching model, these systematics play a small role, while the opposite happens for the conservative Chavarria parametrization. As expected, the background rate is also critical to the success of a neutrino experiment leveraging the Skipper CCD technology. A flat background of 1 kdru would allow for a competitive measurement of the weak mixing angle, while a rate of $10 \mathrm{kdru}$ would be prohibitive. Moreover, if the background follows a $E_{I}^{-1}$ spectral shape, similar to the signal, mitigation becomes of the utmost importance. Finally, our findings also show that the experimental sensitivity is not systematics limited for a $3 \mathrm{~kg}$-year exposure. Therefore, better measurements may be achieved by deploying a larger detector. Our findings show that, under realistic assumptions, the measurement of the weak mixing angle performed by this experimental setup would be competitive with other experiments, including DUNE. This measurement would be one of the very few competitive determinations of the weak mixing angle using neutrinos, which is particularly important given the unsettled $\mathrm{NuTeV}$ discrepancy. Regarding the vIOLETA's time line, different groups are currently working on the technological challenges to integrate thousands of sensors on a system of ten kilograms of active silicon mass. A background measurement campaign has recently started using a high purity germanium detector located $12 \mathrm{~m}$ away from the center of the Atucha II nuclear core. A kilogram scale detector is expected to be deployed within the next four years. We hope our results can be useful to experimentalists as a prioritization guide for maximizing the physics output of a reactor neutrino Skipper CCD experiment.

\section{Acknowledgments}

We thank Ivan Sidelnik for invaluable comments on the manuscript, and we are also grateful to Ricardo Piegaia and Ryan Plestid for useful discussions. SRA would like to thank Fermilab's theory group for the kind hospitality during the first stages of this work. Fermilab is operated by the Fermi Research Alliance, LLC under contract No. DE-AC02-07CH11359 with the United States Department of Energy. SRA acknowledges the support of the Spanish Agencia Estatal de Investigacion and the EU "Fondo Europeo de Desarrollo Regional" (FEDER) through the projects PID2019-108892RB-I00/AEI/10.13039/501100011033 and FPA2016-78645-P; the "IFT Centro de Excelencia Severo Ochoa SEV-2016-0597"; as well as the European Union's Horizon 2020 research and innovation programme under the Marie Sklodowska-Curie grant agreement 690575-InvisiblesPlus. DR acknowledges the support of Agencia Nacional de Promoción de la Investigación, el Desarrollo Tecnológico y la Innovación through grant PICT 2018-02153.

Open Access. This article is distributed under the terms of the Creative Commons Attribution License (CC-BY 4.0), which permits any use, distribution and reproduction in any medium, provided the original author(s) and source are credited. 


\section{References}

[1] D.Z. Freedman, Coherent Neutrino Nucleus Scattering as a Probe of the Weak Neutral Current, Phys. Rev. D 9 (1974) 1389 [INSPIRE].

[2] COHERENT collaboration, Observation of Coherent Elastic Neutrino-Nucleus Scattering, Science 357 (2017) 1123 [arXiv: 1708.01294] [INSPIRE].

[3] CoGeNT collaboration, Results from a Search for Light-Mass Dark Matter with a P-type Point Contact Germanium Detector, Phys. Rev. Lett. 106 (2011) 131301 [arXiv: 1002 .4703] [INSPIRE].

[4] A. Beda, V. Brudanin, V. Egorov, D. Medvedev, V. Pogosov, E. Shevchik, M. Shirchenko, A. Starostin and I. Zhitnikov, Gemma experiment: The results of neutrino magnetic moment search, Phys. Part. Nucl. Lett. 10 (2013) 139.

[5] XENON1T collaboration, The XENON1T Dark Matter Search Experiment, Springer Proc. Phys. 148 (2013) 93 [arXiv:1206.6288] [INSPIRE].

[6] S.E. Holland, D.E. Groom, N.P. Palaio, R.J. Stover and M. Wei, Fully depleted, back-illuminated charge-coupled devices fabricated on high-resistivity silicon, IEEE Trans. Electron Devices 50 (2003) 225.

[7] SENSEI collaboration, Single-electron and single-photon sensitivity with a silicon Skipper CCD, Phys. Rev. Lett. 119 (2017) 131802 [arXiv:1706.00028] [INSPIRE].

[8] G. Fernandez Moroni, J. Estrada, G. Cancelo, S.E. Holland, E.E. Paolini and H.T. Diehl, Sub-electron readout noise in a Skipper CCD fabricated on high resistivity silicon, Exper. Astron. 34 (2012) 43 [arXiv:1106.1839] [INSPIRE].

[9] D. Rodrigues et al., Absolute measurement of the Fano factor using a Skipper-CCD, arXiv:2004.11499 [INSPIRE].

[10] SENSEI collaboration, SENSEI: Direct-Detection Constraints on Sub-GeV Dark Matter from a Shallow Underground Run Using a Prototype Skipper-CCD, Phys. Rev. Lett. 122 (2019) 161801 [arXiv:1901.10478] [INSPIRE].

[11] SENSEI collaboration, SENSEI: Direct-Detection Results on sub-GeV Dark Matter from a New Skipper-CCD, Phys. Rev. Lett. 125 (2020) 171802 [arXiv:2004.11378] [INSPIRE].

[12] DAMIC and DAMIC-M collaborations, Search for low-mass dark matter with the DAMIC experiment, in 16th Rencontres du Vietnam: Theory meeting experiment: Particle Astrophysics and Cosmology, (2020) [arXiv:2003.09497] [INSPIRE].

[13] Oscura, https://astro.fnal.gov/science/dark-matter/oscura/ (2020).

[14] G. Fernandez Moroni, J. Estrada, E.E. Paolini, G. Cancelo, J. Tiffenberg and J. Molina, Charge Coupled Devices for detection of coherent neutrino-nucleus scattering, Phys. Rev. D 91 (2015) 072001 [arXiv: 1405.5761] [INSPIRE].

[15] X.B. Ma, W.L. Zhong, L.Z. Wang, Y.X. Chen and J. Cao, Improved calculation of the energy release in neutron-induced fission, Phys. Rev. C 88 (2013) 014605 [arXiv:1212.6625] [INSPIRE].

[16] CONNIE collaboration, The CONNIE experiment, J. Phys. Conf. Ser. 761 (2016) 012057 [arXiv: 1608.01565] [INSPIRE].

[17] NUCLEUS collaboration, Exploring CENNS with NUCLEUS at the CHOOZ nuclear power plant, Eur. Phys. J. C 79 (2019) 1018 [arXiv: 1905.10258] [INSPIRE]. 
[18] C. Buck et al., A novel experiment for coherent elastic neutrino nucleus scattering: CONUS, J. Phys. Conf. Ser. 1342 (2020) 012094 [inSPIRE].

[19] CONNIE collaboration, Search for light mediators in the low-energy data of the CONNIE reactor neutrino experiment, JHEP 04 (2020) 054 [arXiv: 1910.04951] [INSPIRE].

[20] J.I. Collar et al., Coherent neutrino-nucleus scattering detection with a CsI[Na] scintillator at the SNS spallation source, Nucl. Instrum. Meth. A 773 (2015) 56 [arXiv:1407.7524] [INSPIRE].

[21] W. Skiba and Q. Xia, Electroweak Constraints from the COHERENT Experiment, arXiv: 2007.15688 [INSPIRE].

[22] P. Coloma, I. Esteban, M.C. Gonzalez-Garcia and J. Menendez, Determining the nuclear neutron distribution from Coherent Elastic neutrino-Nucleus Scattering: current results and future prospects, JHEP 08 (2020) 030 [arXiv: 2006. 08624] [INSPIRE].

[23] P. Coloma, M.C. Gonzalez-Garcia, M. Maltoni and T. Schwetz, COHERENT Enlightenment of the Neutrino Dark Side, Phys. Rev. D 96 (2017) 115007 [arXiv:1708.02899] [InSPIRE].

[24] Y. Farzan and M. Tortola, Neutrino oscillations and Non-Standard Interactions, Front. in Phys. 6 (2018) 10 [arXiv:1710.09360] [INSPIRE].

[25] J. Liao and D. Marfatia, COHERENT constraints on nonstandard neutrino interactions, Phys. Lett. B 775 (2017) 54 [arXiv: 1708.04255] [INSPIRE].

[26] I. Esteban, M.C. Gonzalez-Garcia, M. Maltoni, I. Martinez-Soler and J. Salvado, Updated constraints on non-standard interactions from global analysis of oscillation data, JHEP 08 (2018) 180 [Addendum ibid. 12 (2020) 152] [arXiv:1805.04530] [INSPIRE].

[27] D. Aristizabal Sierra, V. De Romeri and N. Rojas, COHERENT analysis of neutrino generalized interactions, Phys. Rev. D 98 (2018) 075018 [arXiv:1806. 07424] [INSPIRE].

[28] W. Altmannshofer, M. Tammaro and J. Zupan, Non-standard neutrino interactions and low energy experiments, JHEP 09 (2019) 083 [arXiv:1812.02778] [INSPIRE].

[29] M. Abdullah, J.B. Dent, B. Dutta, G.L. Kane, S. Liao and L.E. Strigari, Coherent elastic neutrino nucleus scattering as a probe of a Z' through kinetic and mass mixing effects, Phys. Rev. D 98 (2018) 015005 [arXiv: 1803.01224] [InSPIRE].

[30] M.C. Gonzalez-Garcia, M. Maltoni, Y.F. Perez-Gonzalez and R. Zukanovich Funchal, Neutrino Discovery Limit of Dark Matter Direct Detection Experiments in the Presence of Non-Standard Interactions, JHEP 07 (2018) 019 [arXiv: 1803.03650] [INSPIRE].

[31] C. Giunti, General COHERENT constraints on neutrino nonstandard interactions, Phys. Rev. D 101 (2020) 035039 [arXiv: 1909.00466] [INSPIRE].

[32] D. Aristizabal Sierra, V. De Romeri and N. Rojas, CP violating effects in coherent elastic neutrino-nucleus scattering processes, JHEP 09 (2019) 069 [arXiv:1906.01156] [INSPIRE].

[33] I. Bischer and W. Rodejohann, General neutrino interactions from an effective field theory perspective, Nucl. Phys. B 947 (2019) 114746 [arXiv: 1905.08699] [INSPIRE].

[34] B.C. Canas, E.A. Garces, O.G. Miranda, A. Parada and G. Sanchez Garcia, Interplay between nonstandard and nuclear constraints in coherent elastic neutrino-nucleus scattering experiments, Phys. Rev. D 101 (2020) 035012 [arXiv:1911.09831] [INSPIRE].

[35] K.S. Babu, P.S.B. Dev, S. Jana and A. Thapa, Non-Standard Interactions in Radiative Neutrino Mass Models, JHEP 03 (2020) 006 [arXiv: 1907.09498] [INSPIRE]. 
[36] P.B. Denton and J. Gehrlein, A Statistical Analysis of the COHERENT Data and Applications to New Physics, arXiv:2008.06062 [INSPIRE].

[37] L.J. Flores, N. Nath and E. Peinado, Non-standard neutrino interactions in U(1)' model after COHERENT data, JHEP 06 (2020) 045 [arXiv: 2002.12342] [INSPIRE].

[38] J.B. Dent et al., New Directions for Axion Searches via Scattering at Reactor Neutrino Experiments, Phys. Rev. Lett. 124 (2020) 211804 [arXiv: 1912.05733] [INSPIRE].

[39] M. Pospelov, Neutrino Physics with Dark Matter Experiments and the Signature of New Baryonic Neutral Currents, Phys. Rev. D 84 (2011) 085008 [arXiv:1103.3261] [InSPIRE].

[40] R. Harnik, J. Kopp and P.A.N. Machado, Exploring nu Signals in Dark Matter Detectors, JCAP 07 (2012) 026 [arXiv:1202.6073] [INSPIRE].

[41] M. Pospelov and J. Pradler, Elastic scattering signals of solar neutrinos with enhanced baryonic currents, Phys. Rev. D 85 (2012) 113016 [Erratum ibid. 88 (2013) 039904] [arXiv: 1203.0545] [INSPIRE].

[42] D.K. Papoulias and T.S. Kosmas, COHERENT constraints to conventional and exotic neutrino physics, Phys. Rev. D 97 (2018) 033003 [arXiv:1711.09773] [INSPIRE].

[43] Y. Farzan, M. Lindner, W. Rodejohann and X.-J. Xu, Probing neutrino coupling to a light scalar with coherent neutrino scattering, JHEP 05 (2018) 066 [arXiv:1802.05171] [INSPIRE].

[44] C. Bœhm, D.G. Cerdeño, P.A.N. Machado, A. Olivares-Del Campo, E. Perdomo and E. Reid, How high is the neutrino floor?, JCAP 01 (2019) 043 [arXiv:1809.06385] [INSPIRE].

[45] P.B. Denton, Y. Farzan and I.M. Shoemaker, Testing large non-standard neutrino interactions with arbitrary mediator mass after COHERENT data, JHEP 07 (2018) 037 [arXiv: 1804.03660] [INSPIRE].

[46] M. Cadeddu, C. Giunti, K.A. Kouzakov, Y.F. Li, A.I. Studenikin and Y.Y. Zhang, Neutrino Charge Radii from COHERENT Elastic Neutrino-Nucleus Scattering, Phys. Rev. D 98 (2018) 113010 [Erratum ibid. 101 (2020) 059902] [arXiv:1810.05606] [INSPIRE].

[47] J. Billard, J. Johnston and B.J. Kavanagh, Prospects for exploring New Physics in Coherent Elastic Neutrino-Nucleus Scattering, JCAP 11 (2018) 016 [arXiv: 1805.01798] [INSPIRE].

[48] B. Dutta, S. Liao, S. Sinha and L.E. Strigari, Searching for Beyond the Standard Model Physics with COHERENT Energy and Timing Data, Phys. Rev. Lett. 123 (2019) 061801 [arXiv: 1903.10666] [INSPIRE].

[49] M. Cadeddu et al., Constraints on light vector mediators through coherent elastic neutrino nucleus scattering data from COHERENT, JHEP 01 (2021) 116 [arXiv:2008.05022] [INSPIRE].

[50] J. Billard, L.E. Strigari and E. Figueroa-Feliciano, Solar neutrino physics with low-threshold dark matter detectors, Phys. Rev. D 91 (2015) 095023 [arXiv: 1409.0050] [InSPIRE].

[51] D.G. Cerdeño, M. Fairbairn, T. Jubb, P.A.N. Machado, A.C. Vincent and C. Bøehm, Physics from solar neutrinos in dark matter direct detection experiments, JHEP 05 (2016) 118 [Erratum ibid. 09 (2016) 048] [arXiv: 1604.01025] [INSPIRE].

[52] D.W.P.d. Amaral, D.G. Cerdeno, P. Foldenauer and E. Reid, Solar neutrino probes of the muon anomalous magnetic moment in the gauged $\mathrm{U}(1)_{L_{\mu}-L_{\tau}}$, JHEP 12 (2020) 155 [arXiv:2006.11225] [INSPIRE]. 
[53] J.A. Formaggio, E. Figueroa-Feliciano and A.J. Anderson, Sterile Neutrinos, Coherent Scattering and Oscillometry Measurements with Low-temperature Bolometers, Phys. Rev. D 85 (2012) 013009 [arXiv: 1107.3512] [INSPIRE].

[54] A.J. Anderson et al., Measuring Active-to-Sterile Neutrino Oscillations with Neutral Current Coherent Neutrino-Nucleus Scattering, Phys. Rev. D 86 (2012) 013004 [arXiv: 1201.3805] [INSPIRE].

[55] B. Dutta, Y. Gao, R. Mahapatra, N. Mirabolfathi, L.E. Strigari and J.W. Walker, Sensitivity to oscillation with a sterile fourth generation neutrino from ultra-low threshold neutrino-nucleus coherent scattering, Phys. Rev. D 94 (2016) 093002 [arXiv:1511.02834] [INSPIRE].

[56] B.C. Cañas, E.A. Garcés, O.G. Miranda and A. Parada, The reactor antineutrino anomaly and low energy threshold neutrino experiments, Phys. Lett. B 776 (2018) 451 [arXiv: 1708.09518] [INSPIRE].

[57] T.S. Kosmas, D.K. Papoulias, M. Tortola and J.W.F. Valle, Probing light sterile neutrino signatures at reactor and Spallation Neutron Source neutrino experiments, Phys. Rev. D 96 (2017) 063013 [arXiv:1703.00054] [INSPIRE].

[58] C. Blanco, D. Hooper and P. Machado, Constraining Sterile Neutrino Interpretations of the LSND and MiniBooNE Anomalies with Coherent Neutrino Scattering Experiments, Phys. Rev. D 101 (2020) 075051 [arXiv:1901.08094] [InSPIRE].

[59] O.G. Miranda, D.K. Papoulias, O. Sanders, M. Tórtola and J.W.F. Valle, Future CEvNS experiments as probes of lepton unitarity and light-sterile neutrinos, Phys. Rev. D 102 (2020) 113014 [arXiv:2008.02759] [INSPIRE].

[60] Y. Cui, M. Pospelov and J. Pradler, Signatures of Dark Radiation in Neutrino and Dark Matter Detectors, Phys. Rev. D 97 (2018) 103004 [arXiv:1711.04531] [InSPIRE].

[61] S.-F. Ge and I.M. Shoemaker, Constraining Photon Portal Dark Matter with Texono and Coherent Data, JHEP 11 (2018) 066 [arXiv:1710.10889] [INSPIRE].

[62] E. Bertuzzo, S. Jana, P.A.N. Machado and R. Zukanovich Funchal, Dark Neutrino Portal to Explain MiniBooNE excess, Phys. Rev. Lett. 121 (2018) 241801 [arXiv:1807.09877] [INSPIRE].

[63] B. Dutta, D. Kim, S. Liao, J.-C. Park, S. Shin and L.E. Strigari, Dark matter signals from timing spectra at neutrino experiments, Phys. Rev. Lett. 124 (2020) 121802 [arXiv: 1906.10745] [INSPIRE].

[64] vIOLETA Collaboration, The violeta collaboration website, https://www.violetaexperiment.com/.

[65] vIOLETA Collaboration, D. Rodrigues, G.F. Moroni, C. Bonifazi and J.C. D'Olivo, vIOLETA: Neutrino interaction observation with a low energy threshold array, (2020) https://nusoft.fnal.gov/nova/nu2020postersession/pdf/posterPDF-521.pdf.

[66] vIOLETA Collaboration, E. Pozzi, I.P. Sidelnik, L. Galeta and P. Curotto, Short baseline neutrino program in argentina, (2020) https://nusoft.fnal.gov/nova/nu2020postersession/pdf/posterPDF-523.pdf.

[67] vIOLETA Collaboration, I.J. Martinez-Soler, P. Machado, Y.F. Perez-Gonzalez and S. Rosauro-Alcaraz, A first study of the physics potential of a reactor neutrino experiment with skipper-ccds, (2020) https://nusoft.fnal.gov/nova/nu2020postersession/pdf/posterPDF-508.pdf. 
[68] H. Davoudiasl, H.-S. Lee and W.J. Marciano, Muon $g$ - 2, rare kaon decays, and parity violation from dark bosons, Phys. Rev. D 89 (2014) 095006 [arXiv: 1402.3620] [INSPIRE].

[69] COHERENT collaboration, COHERENT 2018 at the Spallation Neutron Source, arXiv: 1803.09183 [INSPIRE].

[70] DAYA BAY collaboration, Extraction of the ${ }^{235} U$ and ${ }^{239} P u$ Antineutrino Spectra at Daya Bay, Phys. Rev. Lett. 123 (2019) 111801 [arXiv:1904.07812] [inSPIRE].

[71] J. Engel, Nuclear form-factors for the scattering of weakly interacting massive particles, Phys. Lett. B 264 (1991) 114 [inSPIRE].

[72] J.D. Lewin and P.F. Smith, Review of mathematics, numerical factors, and corrections for dark matter experiments based on elastic nuclear recoil, Astropart. Phys. 6 (1996) 87 [INSPIRE].

[73] Particle Data Group collaboration, Review of Particle Physics, PTEP 2020 (2020) 083C01 [INSPIRE].

[74] CONNIE collaboration, Exploring low-energy neutrino physics with the Coherent Neutrino Nucleus Interaction Experiment, Phys. Rev. D 100 (2019) 092005 [arXiv:1906.02200] [INSPIRE].

[75] G. Fernandez-Moroni, K. Andersson, A. Botti, J. Estrada, D. Rodrigues and J. Tiffenberg, Charge collection efficiency in back-illuminated Charge-Coupled Devices, arXiv: 2007.04201 [INSPIRE].

[76] M. Bowen and P. Huber, Reactor neutrino applications and coherent elastic neutrino nucleus scattering, Phys. Rev. D 102 (2020) 053008 [arXiv: 2005.10907] [INSPIRE].

[77] SENSEI collaboration, SENSEI: Direct-Detection Constraints on Sub-GeV Dark Matter from a Shallow Underground Run Using a Prototype Skipper-CCD, Phys. Rev. Lett. 122 (2019) 161801 [arXiv:1901.10478] [INSPIRE].

[78] SENSEI collaboration, SENSEI: Direct-Detection Results on sub-GeV Dark Matter from a New Skipper-CCD, Phys. Rev. Lett. 125 (2020) 171802 [arXiv:2004.11378] [INSPIRE].

[79] A.E. Chavarria et al., Measurement of the ionization produced by sub-keV silicon nuclear recoils in a CCD dark matter detector, Phys. Rev. D 94 (2016) 082007 [arXiv:1608.00957] [INSPIRE].

[80] G. Mention et al., The Reactor Antineutrino Anomaly, Phys. Rev. D 83 (2011) 073006 [arXiv:1101.2755] [INSPIRE].

[81] P. Huber, On the determination of anti-neutrino spectra from nuclear reactors, Phys. Rev. C 84 (2011) 024617 [Erratum ibid. 85 (2012) 029901] [arXiv:1106.0687] [INSPIRE].

[82] A.C. Hayes, J.L. Friar, G.T. Garvey, G. Jungman and G. Jonkmans, Systematic Uncertainties in the Analysis of the Reactor Neutrino Anomaly, Phys. Rev. Lett. 112 (2014) 202501 [arXiv: 1309.4146] [INSPIRE].

[83] A.C. Hayes and P. Vogel, Reactor Neutrino Spectra, Ann. Rev. Nucl. Part. Sci. 66 (2016) 219 [arXiv: 1605.02047] [INSPIRE].

[84] A.A. Sonzogni, E.A. McCutchan and A.C. Hayes, Dissecting Reactor Antineutrino Flux Calculations, Phys. Rev. Lett. 119 (2017) 112501 [INSPIRE].

[85] M. Dentler et al., Updated Global Analysis of Neutrino Oscillations in the Presence of eV-Scale Sterile Neutrinos, JHEP 08 (2018) 010 [arXiv: 1803.10661] [INSPIRE]. 
[86] A. Diaz, C.A. Argüelles, G.H. Collin, J.M. Conrad and M.H. Shaevitz, Where Are We With Light Sterile Neutrinos?, Phys. Rept. 884 (2020) 1 [arXiv:1906.00045] [INSPIRE].

[87] S. Böser et al., Status of Light Sterile Neutrino Searches, Prog. Part. Nucl. Phys. 111 (2020) 103736 [arXiv:1906.01739] [INSPIRE].

[88] RENO collaboration, Measurement of Reactor Antineutrino Oscillation Amplitude and Frequency at RENO, Phys. Rev. Lett. 121 (2018) 201801 [arXiv:1806.00248] [inSPIRE].

[89] P. Vogel and J. Engel, Neutrino Electromagnetic Form-Factors, Phys. Rev. D 39 (1989) 3378 [inSPIRE].

[90] Daya BAy collaboration, Improved Measurement of the Reactor Antineutrino Flux and Spectrum at Daya Bay, Chin. Phys. C 41 (2017) 013002 [arXiv: 1607.05378] [INSPIRE].

[91] A. de Gouvêa, P.A.N. Machado, Y.F. Perez-Gonzalez and Z. Tabrizi, Measuring the Weak Mixing Angle in the DUNE Near Detector Complex, Phys. Rev. Lett. 125 (2020) 051803 [arXiv:1912.06658] [INSPIRE].

[92] NuTeV collaboration, A Precise Determination of Electroweak Parameters in Neutrino Nucleon Scattering, Phys. Rev. Lett. 88 (2002) 091802 [Erratum ibid. 90 (2003) 239902] [hep-ex/0110059] [INSPIRE].

[93] W. Loinaz, N. Okamura, T. Takeuchi and L.C.R. Wijewardhana, The NuTeV anomaly, neutrino mixing, and a heavy Higgs boson, Phys. Rev. D 67 (2003) 073012 [hep-ph/0210193] [INSPIRE].

[94] Y. Liang, L.-B. Chen and C.-F. Qiao, X(16.7) as the solution of the NuTeV anomaly, Chin. Phys. C 41 (2017) 063105 [arXiv: 1607.08309] [InSPIRE].

[95] J. Guena, M. Lintz and M.A. Bouchiat, Measurement of the parity violating 6S-7S transition amplitude in cesium achieved within $2 \times 10^{-13}$ atomic-unit accuracy by stimulated-emission detection, Phys. Rev. A 71 (2005) 042108 [physics/0412017] [INSPIRE].

[96] QwEAK collaboration, Precision measurement of the weak charge of the proton, Nature $\mathbf{5 5 7}$ (2018) 207 [arXiv: 1905. 08283] [inSPIRE].

[97] SLAC E158 collaboration, Precision measurement of the weak mixing angle in Moller scattering, Phys. Rev. Lett. 95 (2005) 081601 [hep-ex/0504049] [INSPIRE].

[98] D. Wang et al., Measurement of Parity-Violating Asymmetry in Electron-Deuteron Inelastic Scattering, Phys. Rev. C 91 (2015) 045506 [arXiv:1411.3200] [INSPIRE].

[99] ALEPH, DELPHI, L3, OPAL and SLD collaborations, LEP Electroweak Working Group, SLD Electroweak Group and SLD Heavy Flavour Group, Precision electroweak measurements on the $Z$ resonance, Phys. Rept. 427 (2006) 257 [hep-ex/0509008] [INSPIRE].

[100] SLD collaboration, First measurement of the left-right charge asymmetry in hadronic $Z$ boson decays and a new determination of $\sin ^{2} \theta_{W}^{\text {eff }}$, Phys. Rev. Lett. 78 (1997) 17 [hep-ex/9609019] [INSPIRE].

[101] CDF and D0 collaborations, Tevatron Run II combination of the effective leptonic electroweak mixing angle, Phys. Rev. D 97 (2018) 112007 [arXiv:1801.06283] [INSPIRE].

[102] ATLAS collaboration, Measurement of the forward-backward asymmetry of electron and muon pair-production in pp collisions at $\sqrt{s}=7 \mathrm{TeV}$ with the ATLAS detector, JHEP 09 (2015) 049 [arXiv: 1503.03709] [inSPIRE].

[103] CMS collaboration, Measurement of the weak mixing angle using the forward-backward asymmetry of Drell-Yan events in pp collisions at 8 TeV, Eur. Phys. J. C 78 (2018) 701 [arXiv: 1806.00863] [INSPIRE]. 
[104] LHCb collaboration, Measurement of the forward-backward asymmetry in $Z / \gamma^{*} \rightarrow \mu^{+} \mu^{-}$ decays and determination of the effective weak mixing angle, JHEP 11 (2015) 190 [arXiv: 1509.07645] [INSPIRE].

[105] J. Lindhard, V. Nielsen, M. Scharff and P.V. Thomsen, Integral Equations Governing Radiation Effects, Mat. Fys. Medd. Dan. Vid. Selsk. 33 (1963) 1.

[106] D.M. Mei, Z.B. Yin, L.C. Stonehill and A. Hime, A Model of Nuclear Recoil Scintillation Efficiency in Noble Liquids, Astropart. Phys. 30 (2008) 12 [arXiv:0712.2470] [INSPIRE]. 